\title{
The Gross-Pitaevskii Equation and Bose-Einstein condensates
}

\author{
J. Rogel-Salazar \\ Applied Mathematics and Quantitative Analysis Group, \\ Science and Technology Research Institute, \\ School of Physics Astronomy and Mathematics, \\ University of Hertfordshire, Hatfield, AL10 9AB, U.K. \\ E-mail: j.rogel@physics.org
}

\begin{abstract}
The Gross-Pitaevskii equation is discussed at the level of an advanced course on statistical physics. In the standard literature the Gross-Pitaevskii equation is usually obtained in the framework of the second quantisation formalism, which in many cases goes beyond the material covered in many advanced undergraduate courses. In this paper, we motivate the derivation of the Gross-Pitaevskii equation (GPE) in relationship to concepts from statistical physics, highlighting a number of applications from dynamics of a Bose-Einstein condensate to the excitations of the gas cloud. This paper may be helpful not only in encouraging the discussion of modern developments in a statistical mechanics course, but also can be of use in other contexts such as mathematical physics and modelling. The paper is suitable for undergraduate and graduate students, as well as general physicists.
\end{abstract}

PACS numbers: 01.40.Fk, 02.30.Xx, 05.30.Jp, 03.75.Hh, 03.75.Mn, 67.10.-j

Subjects: Quantum Gases, Statistical Physics, Education, Bose-Einstein Condensation Submitted to: Eur. J. Phys. 


\section{Introduction}

The realisation of Bose-condensed gases with alkali elements [1, 2] provided physicists with a great opportunity to test a new regime of matter that until then was considered a purely theoretical concept. The theoretical basis for the description of these systems has seen the development of excellent reviews [3, 4] and textbooks $[5,6]$. The dynamics of the condensate at zero temperature is generally described with the Gross-Pitaevskii equation (GPE) which is effectively a mean-field approximation for the interparticle interactions. It is usually the case that the GPE is derived from the framework of second quantisation, which may pose some barriers to exposing the description of trapped Bosecondensed gases to newcomers to the field. Since Bose-Einstein condensation (BEC) can be understood from statistical correlations, without the need of boson-boson interaction, there is a feasible possibility of discussing the phenomenon beyond the usual BoseEinstein statistics [7]. With that in mind, the aim of this paper is to enable students and lecturers to approach the description of a BEC in an accessible manner which allows them to tackle more advanced treatments using concepts readily available to them.

\section{Bose-Einstein condensation}

From the description provided by thermodynamics, within a gas, all the particles behave in the same manner and in principle they can occupy certain quantum states, that is certain energy states [8]. If these particles are fermions, two or more of them cannot occupy the same quantum state (i.e. the Pauli exclusion principle). However, if they are bosons, any number of them can occupy the same quantum state. When we put these particles in a given configuration, they will get distributed in the energy levels of such a configuration, with an increasing occupation of the states of minimum energy as we lower the temperature.

For a collection of bosons, and in the limit where the temperature goes to zero, all the particles are going to occupy the ground state of the system. Actually, before achieving this limit, an spectacular accumulation of particles in the ground state can be observed. Therefore, for a sufficiently low temperature, the majority of the particles are in the same quantum state, and have the same velocity. In this way, the collection of bosons behaves like a macroscopic fluid with new properties, such as superfluidity [9].

In order to study these properties, it is only necessary to concentrate on the ground state. The state of the particles in a certain level is described by a normalised function, which is an eigenvector of the Hamiltonian associated to the physical situation under consideration, and whose eigenvalue corresponds to the energy of that level. Thus, we have to find the eigenvectors with minimum eigenvalue for the Hamiltonian that describes our system. This Hamiltonian includes all the interactions between every pair of particles. In other words, if there are $N$ particles in the system, the Hamiltonian will have $N^{2}$ terms. In order to simplify the problem, we make use of the "mean-field" approximation, which means that the action felt by a given particle due to the rest is 
substituted by the mean action of the fluid over the particle. This approximation is good if one can neglect the correlations in the gas, that is, if the gas is diluted, which is the case for the condensates obtained experimentally. In this way, we have changed a complex model for the interaction among bosons by a very simplified one that is valid for diluted gases. We now have to find now an approximate value for the first eigenvalue of this new Hamiltonian by minimising a functional within a restricted class of Ansatz - the functions for which all the bosons are in the same state.

As we mentioned above, the interactions between bosons are not necessary for condensation to take place [7]; however, they play a very important role in the properties of the condensate as we will see later on. In that sense, using the usual Schrödinger equation [8] is not enough; instead we require the use of the Gross-Pitaevskii equation (GPE). Other applications of the GPE include the modelling of superconductors [10] (superconduction is a quantum mechanical effect manifested at low temperatures and it could be interpreted as some sort of superfluidity of electron pairs), also to describe optical vortices [11], that resemble small twisters in a superfluid.

\subsection{A model for superfluids and BECs}

When helium gas (isotope ${ }^{4} \mathrm{He}$ ) is cooled down, it is possible to observe a transition into a liquid phase at a temperature of $4.2 \mathrm{~K}$ and at a pressure of 1 atmosphere. If we continue cooling down the system well under $2.17 \mathrm{~K}$, this liquid phase acquires highly unusual properties: it becomes a superfluid [12].

Superfluidity is manifested, among other effects, by the lack of viscosity; in other words, the liquid flows without friction. Thus, if we launch some of this superfluid helium into a ring-shaped channel for example, it will not stop. If we try to move an object up to a certain velocity across the surface of the liquid, it will not experience any resistance. Another interesting feature of the system is the creation of vortices, which can be seen as small twisters inside the liquid, that behave quite differently from the ones observed in water for example. These vortices have certain quantisation properties (the velocity of the liquid cannot take any arbitrary value).

These properties, observed in liquid helium ${ }^{4} \mathrm{He}$ in 1937, were also seen in helium

${ }^{3} \mathrm{He}$ at a lower temperature and more recently in Bose-Einstein condensates [13]. As discussed above, a BEC is a particular state of matter achieved at ultra cold temperatures. These phenomena are the macroscopic manifestation of quantum effects, and to study them it is therefore required to use quantum mechanics.

In the 1950s, Landau and Ginzburg proposed to model the electrons that give rise to superconductivity as a superfluid with the aid of an equation of the simplified form $[14]$

$$
i \hbar \frac{\partial u}{\partial t}=-\frac{\hbar^{2}}{2} \nabla^{2} u+g u\left(|u|^{2}-1\right),
$$

in the frame of a two-fluid model, where $|u|^{2}$ represents the superfluid density, which flows without friction, whereas the rest of the matter is supposed to be in a normal fluid 
state, and $g$ is a proportionality constant with dimensions of energy. This equation renders satisfactory predictions, but its usage is limited due to the fact that the interactions in a liquid such as helium are fairly strong.

However, the Landau-Ginzburg model is a particular case of an equation that emerges quite naturally when we study the behaviour of Bose-Einstein condensates up to a first order approximation. This equation is known as the Gross-Pitaevskii equation (GPE)

$$
i \hbar \frac{\partial \psi}{\partial t}=\left(-\frac{\hbar^{2}}{2 m} \nabla^{2}+V_{e x t}+g|\psi|^{2}\right) \psi
$$

where $m$ is the mass of the atoms of the condensate, $|\psi|^{2}$ is the atomic density, $V_{\text {ext }}$ represents an external potential and $g$ is a parameter that measures the atomic interactions. The GPE has the same mathematical form as the nonlinear Schrödinger equation (NLSE), which is basically the Schrödinger equation

$$
i \hbar \frac{\partial \psi}{\partial t}=\left(-\frac{\hbar^{2}}{2 m} \nabla^{2}+V_{e x t}\right) \psi
$$

plus a nonlinear term that in this case takes into account the interaction between the particles. In the case of Bose-Eintein condensates, the interactions are so weak that the predictions made with this equation are very good.

In the following section we will derive the GPE and will use it to present some of the problems that have been studied within the mean-field approximation and which can be used as examples to follow up discussions in undergraduate and graduate lectures related to this subject such as statistical physics, quantum mechanics or mathematical physics. We will study the simple case of a fluid in one dimension, and we will show that if the bosons have an attractive scattering length, the fluid is no longer stable for certain sizes of the system. We will also address the excitations in the Bose-condensed gas.

\section{The Gross-Pitaevskii equation}

As we have discussed above, a Bose-Einstein condensate is obtained from a collection of bosons in the ground state at very low temperatures. We can therefore ask about the energy of the ground state and use this to provide us with information about the system as we can indeed do for any other gas. The general Hamiltonian that describes the system is given by

$$
\hat{H}=\sum_{i=1}^{N}\left(\frac{\mathbf{p}_{i}^{2}}{2 m}+V_{\text {ext }}\left(\mathbf{r}_{i}\right)\right)+\frac{1}{2} \sum_{i=1}^{N} \sum_{j \neq i}^{N} V\left(\left|\mathbf{r}_{i}-\mathbf{r}_{j}\right|\right),
$$

where the first term on the right-hand-side is the kinetic energy energy of the $i$-th particle, the following term represents the external effects introduced by the trapping potential $V_{\text {ext }}$, and the final term represents the interactions between the $N$ particles. The ground state corresponds to the minimum energy and thus we can find it by 
minimising it. In that respect, it is therefore convenient to use the concept of the thermodynamic potentials which are useful in determining the equilibrium state of a system not in isolation and are usually introduced early on in undergraduate courses on thermodynamics and thermal physics $[8,15]$. Using the free energy, we have that we need to minimise $F=E-\mu N$, where $E$ is the energy and $\mu$ is the chemical potential.

Given a Hamiltonian $\hat{H}$ and a wavefunction $\psi$, we can obtain the energy as follows:

$$
E(\psi)=\frac{\langle\psi|\hat{H}| \psi\rangle}{\langle\psi \mid \psi\rangle}
$$

and as such we can use this expression to minimise the free energy $F$. In the condensate we have $N$ particles and we can thus associate a wavefunction $\psi_{i}$ to every one of them. However, in order to capture the essential aspects of the problem we resort to making a mean-field approximation. This means that for one particle, all the rest have the same status as they all are in the same independent state $|\psi\rangle$ and thus we can drop the labelling of the wavefunctions. In that way we need to minimise the free energy over a space of functions of the type $|\Psi\rangle=|\psi\rangle \otimes|\psi\rangle \otimes \cdots \otimes|\psi\rangle$, where $\otimes$ represents the tensor product and thus $|\Psi\rangle$ is the $N$-particle tensor product wavefunction; we are considering the following normalisation $\langle\Psi \mid \Psi\rangle=1$. This approximation is valid if the condensate is not very dense; otherwise, the interactions with the closer neighbours would be much stronger than with the particles that are farther apart.

Our problem is thus reduced to minimising $F(\Psi)=\langle\Psi|\hat{H}| \Psi\rangle-\mu\langle\Psi \mid \Psi\rangle$. Let us now compute each of the terms involved in this calculation. For the kinetic energy term, we have that

$$
\begin{aligned}
\left\langle\Psi\left|\sum_{i=1}^{N} \frac{\mathbf{p}^{2}}{2 m}\right| \Psi\right\rangle & =\sum_{i=1}^{N} \frac{\hbar^{2}}{2 m} \int \nabla \psi^{*}\left(\mathbf{r}_{i}\right) \nabla \psi\left(\mathbf{r}_{i}\right) d \mathbf{r}_{i}, \\
& =N \frac{\hbar^{2}}{2 m} \int|\nabla \psi(\mathbf{r})|^{2} d \mathbf{r} \\
& =-N \frac{\hbar^{2}}{2 m} \int \psi^{*}(\mathbf{r}) \nabla^{2} \psi(\mathbf{r}) d \mathbf{r},
\end{aligned}
$$

where as stated above $|\Psi\rangle$ is the $N$-particle tensor product wavefunction and $\psi(\mathbf{r})$ is the single particle wavefunction; we have used Green's identity for obtaining the last line in equation (6) [16]. The potential term can readily be written as

$$
\left\langle\Psi\left|\sum_{i=1}^{N} V_{e x t}\left(\mathbf{r}_{i}\right)\right| \Psi\right\rangle=N \int \psi^{*}(\mathbf{r}) V_{\text {ext }} \psi(\mathbf{r}) d \mathbf{r} .
$$

For the interaction term we have that

$$
\begin{aligned}
& \left\langle\Psi\left|\frac{1}{2} \sum_{i=1}^{N} \sum_{j \neq i}^{N} V\left(\left|\mathbf{r}_{i}-\mathbf{r}_{j}\right|\right)\right| \Psi\right\rangle \\
& =\frac{1}{2} \sum_{i=1}^{N} \sum_{j \neq i}^{N} \int d \mathbf{r}_{i} \int \psi^{*}\left(\mathbf{r}_{i}\right) \psi^{*}\left(\mathbf{r}_{j}\right) V\left(\left|\mathbf{r}_{i}-\mathbf{r}_{j}\right|\right) \psi\left(\mathbf{r}_{i}\right) \psi\left(\mathbf{r}_{j}\right) d \mathbf{r}_{j}, \\
& =\frac{N(N-1)}{2} \int d \mathbf{r} \int \psi^{*}(\mathbf{r}) \psi\left(\mathbf{r}^{\prime}\right) V\left(\left|\mathbf{r}-\mathbf{r}^{\prime}\right|\right) \psi(\mathbf{r}) \psi\left(\mathbf{r}^{\prime}\right) d \mathbf{r}^{\prime}
\end{aligned}
$$


Finally, for the last term in the free energy we have that

$$
\mu\langle\Psi \mid \Psi\rangle=\mu\left(\int \psi^{*}(\mathbf{r}) \psi(\mathbf{r}) d \mathbf{r}\right)^{N},
$$

where we have written the expression in order to facilitate the rest of our calculations.

Given the expressions above, we now need to minimise them. This can be done following a procedure based on calculus of variations $[17,18]$. In other words, we will consider a small variation in the wavefunction $\psi(\mathbf{r})$, but instead of varying its real and imaginary parts, we take $\psi$ and $\psi^{*}$ as independent variables. In that way, we can readily obtain the functional derivatives $\frac{\delta \ldots}{\delta \psi^{*}}$ for expressions (6) and (7). In the case of the interaction term, expression (8), we have contributions from each of the two $\psi^{*}$, but the $\mathbf{r}$ can be permuted, which results in the following expression

$$
\begin{aligned}
& \frac{\delta}{\delta \psi^{*}}\left\langle\Psi\left|\frac{1}{2} \sum_{i=1}^{N} \sum_{j \neq i}^{N} V\left(\left|\mathbf{r}_{i}-\mathbf{r}_{j}\right|\right)\right| \Psi\right\rangle \\
& =N(N-1) \int \delta \psi^{*}(\mathbf{r})\left(\int|\psi(\mathbf{r})|^{2} V\left(\left|\mathbf{r}-\mathbf{r}^{\prime}\right|\right) d \mathbf{r}^{\prime}\right) \psi(\mathbf{r}) d \mathbf{r} .
\end{aligned}
$$

Similarly, for the chemical potential we have that

$$
\begin{aligned}
\frac{\delta\langle\Psi \mid \Psi\rangle}{\delta \psi^{*}} & =N\left(\int \psi^{*}(\mathbf{r}) \psi(\mathbf{r}) d \mathbf{r}\right)^{N-1} \int \delta \psi^{*}(\mathbf{r}) \psi(\mathbf{r}) d \mathbf{r} \\
& =N \int \delta \psi^{*}(\mathbf{r}) \psi(\mathbf{r}) d \mathbf{r} .
\end{aligned}
$$

Putting together all the different terms for the free energy $F$, we have that the variation is given by:

$$
\begin{aligned}
\frac{\delta F}{\delta \psi^{*}}=0 & =N \int\left[-\frac{\hbar^{2}}{2 m} \nabla^{2} \psi(\mathbf{r})+V_{\text {ext }}(\mathbf{r}) \psi(\mathbf{r})\right. \\
& \left.+(N-1)\left(\int|\psi(\mathbf{r})|^{2} V\left(\left|\mathbf{r}-\mathbf{r}^{\prime}\right|\right) d \mathbf{r}^{\prime}\right) \psi(\mathbf{r})-\mu \psi(\mathbf{r})\right] \delta \psi^{*}(\mathbf{r}) d \mathbf{r},
\end{aligned}
$$

and therefore the quantity inside the square brackets in the above expression must vanish. It is quite common to choose an interaction potential such that $V\left(\left|\mathbf{r}-\mathbf{r}^{\prime}\right|\right)=$ $\frac{4 \pi \hbar^{2}}{m} a \delta\left(\mathbf{r}-\mathbf{r}^{\prime}\right)$ where $a$ is the $s$-wave scattering length and using the approximation that $N-1 \simeq N$ we end up with

$$
-\frac{\hbar^{2}}{2 m} \nabla^{2} \psi(\mathbf{r})+V_{e x t}(\mathbf{r}) \psi(\mathbf{r})+N \frac{4 \pi \hbar^{2}}{m} a|\psi(\mathbf{r})|^{2} \psi(\mathbf{r})=\mu \psi(\mathbf{r})
$$

which is the time-independent Gross-Pitaevskii equation. The scattering length $a$ measures the intensity of the interactions between the bosons. Its sign indicates whether the interactions are attractive $(a<0)$ or repulsive $(a>0)$; we will later discuss the importance of this distinction. In this way, the minimisation of equation (5) corresponds to the minimisation of the free energy $F=E-\mu N$, which is a well-known result of statistical physics. 


\subsection{Some comments about the GPE}

As we mentioned above, the function $\psi$ is a complex valued function. In that respect, following the standard interpretation in quantum mechanics, its square modulus corresponds to a probability density; given the normalisation condition $\langle\Psi \mid \Psi\rangle=1$, this quantity is the probability density of finding a boson. We can equally choose the normalisation such that $\langle\Psi \mid \Psi\rangle=N$, in this case the square modulus represents the number density of the fluid.

The phase does not have an intrinsic meaning but its fluctuations do. Let us write the wavefunction as $\psi=\sqrt{\rho} \exp (i \theta)$ and as such the probability current density can be expressed as:

$$
\mathbf{j}=\rho \mathbf{v}=\frac{i \hbar}{2 m}\left(\psi \nabla \psi^{*}-\psi^{*} \nabla \psi\right),
$$

where $\rho$ is the number density and

$$
\mathbf{v}=\frac{\hbar}{m} \nabla \theta
$$

is the velocity of the bosons. We will come back to this point in Section 4.2 where we present the hydrodynamic form of the GPE.

The external potential $V_{\text {ext }}$ allows us to model the action of the external world on the condensate, in particular of a trap. Most of the time, the bosons are confined in a small region of space (by optical and/or magnetic means). This situation is simulated with the aid of a trapping potential with a minimum in the central part. In general any (regular) potential with a minimum can be locally approximated by a harmonic potential, and such that the condensate remains in the part where the quadratic term is dominant. Therefore,

$$
V_{\text {ext }}(\mathbf{r})=\frac{1}{2} m \omega^{2} r^{2}
$$

where $m$ is the mass of the bosons, and $\omega$ corresponds to the frequency of the trap.

In order to provide an idea of the orders of magnitude involved, we summarise in Table 1 some data taken from real experiments [19, 20].

\begin{tabular}{|c||c|c|}
\hline Quantity & Attractive condensate: ${ }^{7} \mathrm{Li}$ & Repulsive condensate ${ }^{87} \mathrm{Rb}$ \\
\hline$m$ & $1.16 \times 10^{-26} \mathrm{~kg}$ & $1.45 \times 10^{-25} \mathrm{~kg}$ \\
\hline$a$ & $-23.3 a_{0}$ & $109 a_{0}$ \\
\hline$\omega$ & $908.41 \mathrm{rad} \cdot \mathrm{s}^{-1}$ & $674.20 \mathrm{rad} \cdot \mathrm{s}^{-1}$ \\
\hline Typical size $r_{0}$ & $3.16 \mu \mathrm{m}$ & $1.04 \mu \mathrm{m}$ \\
\hline$N$ & $\leq 1400$ & 100000 \\
\hline
\end{tabular}

Table 1. Data related to experimental realisations of Bose-Einstein condensation. In the table, $a_{0}=0.529 \times 10^{-10} \mathrm{~m}$ is the Bohr radius.

In the case of rubidium atoms, if the bosons did not interact, the ground state of the trapping potential on its own would be given by $\psi(\mathbf{r}) \propto \mathrm{e}^{-\left(r / r_{0}\right)^{2}}$, where 
$r_{0} \simeq \sqrt{\frac{\hbar}{m \omega}} \simeq 1.04 \mu \mathrm{m}$. However, in the condensates obtained experimentally, the form of the ground state is parabolic. If the condensates are relatively dense, the kinetic energy would be negligible compared to the rest of the terms, and therefore we have that

$$
\psi(\mathbf{r})=\sqrt{\frac{\mu-m \omega^{2} r^{2} / 2}{g N},}
$$

where $g=\frac{4 \pi \hbar^{2}}{m} a$. From the above expression we can see that the condensate has a parabolic form. The physics behind the idea of neglecting the kinetic energy term is that the energy to add a particle at any point in the cloud is the same everywhere. This energy is given by the sum of the external potential $V_{\text {ext }}(\mathbf{r})$ and an interaction contribution, i.e. $g N|\psi(\mathbf{r})|^{2}$. This should be equal to the chemical potential of a uniform gas having a density equal to the local density $\rho=|\psi(\mathbf{r})|^{2}[5]$. In this context, this approximation is referred to as the Thomas-Fermi approximation $[3,21]$ due to its similarity to the Thomas-Fermi approximation used for fermions [22].

\section{The time-dependent GPE}

The GPE that we have already established is related to the stationary state of the condensate. Experimentally, one puts the atoms in favourable conditions for the formation of a condensate, and waits for the bosons to rearrange in a form such that the GPE is valid. However, one might be interested to see what happens when the environment of the condensate evolves or when the bosonic cloud is unstable. In order to do that we need to establish a mean-field equation that will govern the evolution of bosons in the minimum energy level.

According to quantum mechanics, bosons should obey the Schrödinger equation $i \hbar \partial_{t} \Psi=\hat{H} \Psi$, where $\hat{H}$ is the Hamiltonian, analogous to the one given by equation (4). In a similar way, we can consider that $\psi$ (describing the state of the bosons) satisfies a principle of minimum action: the action $\int_{t_{1}}^{t_{2}} \mathrm{~d} t \int \mathrm{d}^{3} \mathbf{r} L$, where $L$ is the Lagrangian density, must be stationary. The Lagrangian density is defined as

$$
L=i \frac{\hbar}{2}\left(\Psi^{*} \partial_{t} \Psi-\Psi \partial_{t} \Psi^{*}\right)-\frac{\hbar^{2}}{2 m}\left(\nabla \Psi^{*}\right) \cdot(\nabla \Psi)-V_{e x t} \Psi^{*} \Psi,
$$

for a single particle. Once we have written the Lagrangian density for our problem, we have to simplify it using functions of the form $|\Psi\rangle=|\psi\rangle \otimes|\psi\rangle \otimes \cdots \otimes|\psi\rangle$ that give a stationary action, and thus will finally lead to equation (2).

It can be verified that equation (13), which does not include the temporal derivative, is a particular case of the time-dependent GPE. We can therefore say that the solutions of equation (13) correspond to stationary states of the gas. 


\subsection{Further remarks}

Let $\tilde{\psi}$ be a solution of the GPE obtained using the normalisation condition that $\langle\tilde{\psi} \mid \tilde{\psi}\rangle=N(\tilde{\psi})$ (refer to Section 3.1). If we multiply equation (2) by $\tilde{\psi}^{*}$, we get

$$
i \hbar \frac{\partial \tilde{\psi}}{\partial t} \tilde{\psi}^{*}=-\frac{\hbar^{2}}{2 m} \nabla^{2} \tilde{\psi} \tilde{\psi}^{*}+V_{e x t}|\tilde{\psi}|^{2}+g|\tilde{\psi}|^{4}-\mu|\tilde{\psi}|^{2}
$$

Writing a similar equation for $\tilde{\psi}^{*}$ and adding them together we obtain

$$
2 i \frac{\partial}{\partial t} N(\tilde{\psi})=\int \mathrm{d}^{3} \mathbf{r}\left[\frac{\hbar^{2}}{2 m}|\nabla \tilde{\psi}|^{2}+\frac{m \omega^{2}}{2}|\mathbf{r}|^{2}|\tilde{\psi}|^{2}+\frac{g}{2}|\tilde{\psi}|^{4}\right]-\mu N(\tilde{\psi}) .
$$

The left-hand side of $(20)$ is an imaginary quantity $(N(\tilde{\psi}) \in \mathbb{R})$, whereas the right-hand side is real, with $N(\tilde{\psi})$ being constant and $\mu$ is fixed from the beginning, therefore the quantity

$$
E=\int \mathrm{d}^{3} \mathbf{r}\left[\frac{\hbar^{2}}{2 m}|\nabla \tilde{\psi}|^{2}+\frac{m \omega^{2}}{2}|\mathbf{r}|^{2}|\tilde{\psi}|^{2}+\frac{g}{2}|\tilde{\psi}|^{4}\right]
$$

must be conserved; it corresponds then to the total energy of the bosonic gas. In this way, a solution of the GPE has two conserved quantities, the number of particles $N$ and the energy $E$ of the system. The existence of these two quantities tells us some things about the eventual divergence of the time-dependent solutions: the norm of $\tilde{\psi}$ is bounded, but the norm of $\nabla^{2} \tilde{\psi}$ will diverge. The eventual explosion of the solutions will be translated by the appearance of peaks with infinite slope.

\subsection{Hydrodynamic form of the GPE}

In general, we can use the time-dependent GPE as shown in equation (2), nonetheless an equivalent set of equations can help us shed light on other properties of the condensate. As pointed out in Section 3.1, for a solution of the form $\psi=\sqrt{\rho} \exp (i \theta)$, one can use the density $\rho$ and the gradient of its phase to describe the system.

With that in mind, let us derive a continuity equation by multiplying the timedependent GPE shown in equation (2) by $\psi^{*}$ and subtracting the complex conjugate of the resulting equation. We end up with the following expression:

$$
\frac{\partial|\psi|^{2}}{\partial t}+\nabla \cdot\left[\frac{i \hbar}{2 m}\left(\psi \nabla \psi^{*}-\psi^{*} \nabla \psi\right)\right]=0
$$

The equation above has the form of a continuity equation for the particle density $\rho$ and can be expressed as follows:

$$
\frac{\partial \rho}{\partial t}+\nabla \cdot(\rho \mathbf{v})=0
$$

where $\mathbf{v}$ is the velocity of the condensate and it is defined by:

$$
\mathbf{v}=\frac{i \hbar}{2 m} \frac{\left(\psi \nabla \psi^{*}-\psi^{*} \nabla \psi\right)}{|\psi|^{2}}=\frac{\hbar}{m} \nabla \theta
$$


Equation (24) tells is that the motion of the condensate corresponds to that of a flow, since the velocity corresponds to the gradient of a scalar quantity. If the phase $\theta$ is not singular we can immediately see that the motion of the condensate is irrotational:

$$
\nabla \times \mathbf{v}=\frac{\hbar}{m} \nabla \times \nabla \theta=0 .
$$

Please note that the result above is only valid when $\theta$ is not singular; this would not be the case at the core of a vortex line. In that case, the single-valuedness of the condensate wavefunction implies that around a closed contour its change in phase, $\Delta \theta$, must be a multiple of $2 \pi$ :

$$
\Delta \theta=\oint \nabla \theta \cdot d \mathbf{l}=2 \pi l
$$

where $l$ is an integer. In that way, the circulation $\Gamma$ around a closed contour is given by:

$$
\Gamma=\oint \mathbf{v} \cdot d \mathbf{l}=\frac{\hbar}{m} 2 \pi l .
$$

In the case of having a singularity in the phase, equation (27) shows that the circulation is quantised.

So far, equation (23) provides us with the equation of motion for $\rho$. Let us obtain an equation of motion for the phase $\theta$. Substituting a wavefunction of the form $\psi=\sqrt{\rho} \exp (i \theta)$ into equation (2), we can then identify the real and imaginary parts of the equation. From this procedure it can be shown that the imaginary part of the equation results in equation (23), whereas the real part is given by:

$$
-\hbar \frac{\partial \theta}{\partial t}=-\frac{\hbar}{2 m \sqrt{\rho}} \nabla \sqrt{\rho}+\frac{1}{2} m \mathbf{v}^{2}+V_{e x t}+g \rho .
$$

Taking the gradient of equation (28) we have that

$$
m \frac{\partial \mathbf{v}}{\partial t}+\frac{1}{2} m \nabla \mathbf{v}^{2}=\frac{\hbar^{2}}{2 m} \nabla\left(\frac{1}{\sqrt{\rho}} \nabla^{2} \sqrt{\rho}\right)-\nabla V_{e x t}-g \nabla \rho,
$$

where we have used equation (24) to write the expression in terms of the velocity v. For an irrotational flow, given that $\frac{1}{2} \nabla \mathbf{v}^{2}=(\mathbf{v} \cdot \nabla) \mathbf{v}+\mathbf{v} \times(\nabla \times \mathbf{v})$, we obtain an equation analogous to the Navier-Stokes equation without viscosity or Euler equation:

$$
m \frac{\partial \mathbf{v}}{\partial t}+m(\mathbf{v} \cdot \nabla) \mathbf{v}=\frac{\hbar^{2}}{2 m} \nabla\left(\frac{1}{\sqrt{\rho}} \nabla^{2} \sqrt{\rho}\right)-\nabla V_{e x t}-g \nabla \rho .
$$

Equations (23) and (29) are exactly equivalent to the GPE, and thus it is possible to use the latter to investigate the behaviour of the fluid. The GPE is, from a mathematical point of view, equivalent to the equations obtained from fluid mechanics. In the case of a condensate with non-singular phase, the BEC behaves like a fluid without viscosity and with irrotational flux. 


\section{Application of the GPE to a cloud of bosons in one dimension}

In this section, we demonstrate the application of the GPE to a relatively simple case: a bosonic gas in 1 dimension in a uniform potential. Such a gas can be obtained by getting a condensate in an elongated trap. If the temperature is low enough, the thermal agitation energy $k_{B} T$ will not exceed the energy threshold of the trap $(\hbar \omega / 2)$ in the directions perpendicular to the axis of the trap and they could be neglected. The case of the elongated trap corresponds to looking for a solution over all space, and we are particularly interested in the phenomena happening in the centre of the trap, where the density is relatively uniform or, in other words, where the condensate behaves as a fluid.

In order to tackle this problem, let us consider a condensate confined by a box with infinitely hard walls. At the wall the wavefunction must vanish; and inside it, the condensate density approaches its bulk. Let us assume that the potential vanishes for $x \leq 0$ and infinite for $x<0$. In the $y$ and $z$ directions the wavefunction is uniform, and thus equation (13) takes the following form:

$$
\frac{-\hbar^{2}}{2 m} \frac{\mathrm{d}^{2} \psi}{\mathrm{d} x^{2}}+U_{0}|\psi|^{2} \psi=\mu \psi
$$

where we have written $U_{0}=N \frac{4 \pi \hbar^{2}}{m} a$. In the case of a uniform gas, it can be seen that the chemical potential is given by $\mu=U_{0}\left|\psi_{0}\right|^{2}$, where $\psi_{0}$ is the wavefunction far from the wall. The equation then becomes

$$
\frac{-\hbar^{2}}{2 m} \frac{\mathrm{d}^{2} \psi}{\mathrm{d} x^{2}}=U_{0}\left(|\psi|^{2}-\left|\psi_{0}\right|^{2}\right) \psi
$$

When we consider repulsive interactions, i.e. $U_{0}>0$, this equation, subject to the boundary conditions $\psi(0)=0$ and $\psi(\infty)=\psi_{0}$, has the solution

$$
\psi(x)=\psi_{0} \tanh \left(\frac{x}{\xi \sqrt{2}}\right)
$$

where $\xi^{2}=\frac{\hbar^{2}}{2 m\left|\psi_{0}\right|^{2} U_{0}}$; this quantity describes the distance over which the wavefunction tends to its bulk value when subjected to local perturbations, and it is therefore referred to as the healing length [4].

In the case of attractive interactions $\left(U_{0}=-\left|U_{0}\right|\right)$ the solution is given by

$$
\psi(x)=\sqrt{2} \psi_{0} \operatorname{sech}\left(\frac{x}{\xi}\right) .
$$

In contrast to the case with repulsive interactions, the density vanishes at large distances from the centre. Since $U_{0}<0$, the interaction energy decreases with increasing density, and thus the condensate collapses [23]. Taking into account the trapping potential, it is possible to obtain metastable condensates as has been shown by Bradley et al. [24].

\subsection{Excitations}

It is possible to linearise the GPE [25] or its hydrodynamic form [26]. In the latter case, we set $\rho=\rho_{0}+\delta \rho$, where $\rho_{o}=\frac{\mu-V}{a}$, and $\mathbf{v}=\delta \mathbf{v}$ and substitute into equations (23) and 
(29). In the case of equation (29), written in a normalised formalism and keeping first order terms only, we have that

$$
\frac{\partial \delta \mathbf{v}}{\partial t}+a \nabla \delta \rho-\frac{1}{4 \rho_{0}} \nabla \nabla^{2} \delta \rho=0 .
$$

Taking the divergence of this equation:

$$
\frac{\partial \nabla \cdot \delta \mathbf{v}}{\partial t}+a \nabla^{2} \delta \rho-\frac{1}{4 \rho_{0}} \nabla^{2} \nabla^{2} \delta \rho=0 .
$$

From equation (23), keeping only first order terms, we have

$$
\frac{\partial \delta \rho}{\partial t}+\rho_{0} \nabla \cdot \delta \mathbf{v}=0
$$

Finally, combining these two expressions, we get the following equation

$$
-\frac{\partial^{2} \delta \rho}{\partial t^{2}}+a \rho_{0} \nabla^{2} \delta \rho-\frac{1}{4} \nabla^{2} \nabla^{2} \delta \rho=0
$$

which can be seen as the equation of propagation of sound waves in the fluid. It corresponds to the dispersion relation, obtained by taking the Fourier transform of $\rho$, namely

$$
\omega^{2}=\mu k^{2}+\frac{k^{4}}{4}
$$

with $\mu<0$ and $a<0$. We can therefore verify the existence of a critical value for the wavenumber $k$ : if $k<\sqrt{-2 \mu}$, then $\omega^{2}<0$ and the periodic perturbations of the wavenumber tend to diverge from the stable solution (there is an explosion). If on the contrary, $k \geq \sqrt{-2 \mu}$, the perturbed solutions oscillate around the constant solution.

\section{Conclusion}

In this paper we have derived the Gross-Pitaevskii equation using concepts covered in many advanced undergraduate courses. The discussion was motivated from the point of view of the behaviour of a gas as explained from results of statistical mechanics. We have seen how a many-body problem in quantum mechanics can be solved using suitable approximations and using a nonlinear partial differential equation which gives relatively good results. Even if the Gross-Pitaevskii equation seems somewhat simple, it can be applied to obtain information about a great number of physical systems such as Bose-Einstein condensates, superfluids or light phenomena. In particular, in this paper we have made use of the GPE to model the ground state of a Bose-Einstein condensate as well as starting to address its excitations in a low-temperature limit. We expect that this paper encourages discussions of modern developments in physics within the context of advanced coursed in statistical mechanics, mathematical physics or physical modelling.

\section{Acknowledgements}

The author would like to thank Dr James Collett for reading and commenting on the manuscript. 


\section{References}

[1] M. H. Anderson, J. R. Ensher, M. R. Matthews, C. E. Wieman, and E. A. Cornell. Observation of Bose-Einstein condensation in a dilute atomic vapor. Science, 269:198, 1995.

[2] K. B. Davis, M.-O. Mewes, M. R. Andrews, N. J. van Druten, D. S. Durfee, D. M. Kurn, and W. Ketterle. Bose-Einstein Condensation in a Gas of Sodium Atoms. Phys. Rev. Lett., 75:3969, 1995.

[3] F. Dalfovo, S. Giorgini, L. P. Pitaevskii, and S. Stringari. Theory of Bose-Einstein condensation in trapped gases. Rev. Mod. Phys., 71:463, 1999.

[4] A. Leggett. Bose-Einstein condensation in the alkali gases: Some fundamental concepts. Rev. Mod. Phys., 73:307-356, Apr 2001.

[5] C. J. Pethick and H. Smith. Bose-Einstein Condensation in Dilute Gases. Cambridge University Press, Cambridge, 2002.

[6] A. Griffin, T. Nikuni, and E. Zaremba. Bose-Condensed Gases at Finite Temperatures. Cambridge University Press, New York, 2009.

[7] L. Ferrari. Approaching Bose-Einstein condensation. Eur. J. Phys., 32:1547, 2011.

[8] R. P. H. Gasser and W. G. Richards. An Introduction to Statistical Thermodynamics. World Scientific, 1995.

[9] L. D. Landau, E. M. Lifshitz, and L. P. Pitaevskii. Statistical Physics: Theory of the Condensed State. Course of Theoretical Physics. Butterworth-Heinemann, 1980.

[10] S. De Palo, C. Castellani, C. Di Castro, and B. K. Chakraverty. Effective action for superconductors and BCS-Bose crossover. Phys. Rev. B, 60:564-573, Jul 1999.

[11] D. Rozas, C. T. Law, and G. A. Swartzlander Jr. Propagation dynamics of optical vortices. J. Opt. Soc. Am. B, 14:3054, 1997.

[12] P. Nozières and D. Pines. The Theory of Quantum Liquids: Superfluid Bose liquids. Advanced Book Classics. Addison-Wesley, 1990.

[13] S. Balibar. The Discovery of Superfluidity. J. of Low Temp. Phys., 146(5/6):441, 207.

[14] V. L. Ginzburg and L. D. Landau. On the Theory of Superconductivity. Zh. Eksperim. I. Teor. Fiz., 20:1060, 1950. Collected papers of L. D. Landau, Pergamon Press (1965), 546.

[15] K Huang. Statistical Mechanics. John Wiley and Sons, 2nd ed. edition, 1987.

[16] W. Kaplan. Advanced Calculus. Addison-Wesley, Advanced Book Program, 1991.

[17] G.M. Ewing. Calculus of Variations with Applications. Dover Books on Mathematics. Dover Publications, 1985.

[18] C. Fox. An Introduction to the Calculus of Variations. Dover Books on Mathematics. Dover Publications, 2010.

[19] C. Huepe, S. Métens, G. Dewel, P. Borckmans, and M.-E. Brachet. Dacay rates in attractive Bose-Einstein condensates. Phys. Rev. Lett., 82:1616, 1999.

[20] J. Soding, D. Guéry-Odelin, P. Desbiolles, F. Chevy, H. Inamori, and J. Dalibard. Three-body decay of a rubidium Bose-Einstein condensate. Preprint cond-mat/9811339.

[21] B. Gordon and C. J. Pethick. Ground-state properties of magnetically trapped bose-condensed rubidium gas. Phys. Rev. Lett., 76:6-9, 1996.

[22] W. Greiner. Quantum Mechanics: Special Chapters. Theoretische Physik. Springer, 1998.

[23] P. A. Ruprecht, M. J. Holland, K. Burnett, and Mark Edwards. Time-dependent solution of the nonlinear schrödinger equation for bose-condensed trapped neutral atoms. Phys. Rev. A, 51:4704-4711, 1995.

[24] C. C. Bradley, C. A. Sackett, and R. G. Hulet. Bose-einstein condensation of lithium: Observation of limited condensate number. Phys. Rev. Lett., 78:985-989, 1997.

[25] C. Cohen-Tannoudji and D. Guéry-Odelin. Advances In Atomic Physics: An Overview. World Scientific, 2011.

[26] C. Nore, M. Abid, and M.-E. Brachet. Decaying Kolmogorov turbulence in a model of superflow. Phys Fluids, 9:2644, 1997. 